\title{
Clinical Characteristics and Outcomes of Oral Mucositis Associated With Immune Checkpoint Inhibitors in Patients With Cancer
}

\author{
Jake S. Jacob, MD; Barbara E. Dutra, MD²; Victor Garcia-Rodriguez, MD²; Kavea Panneerselvam, MD; \\ Fiyinfoluwa O. Abraham, $\mathrm{MD}^{2}$; Fangwen Zou, MD, ${ }^{3,4}$ Weijie Ma, MD ${ }^{3,5}$; Petros Grivas, MD, $\mathrm{PhD}^{6}$; John A. Thompson, $\mathrm{MD}^{6}$; \\ Mehmet Altan, MD; Isabella C. Glitza Oliva, MD, $\mathrm{PhD}^{8}$; Hao Chi Zhang, $\mathrm{MD}^{3}$; Anusha S. Thomas, $\mathrm{MD}^{3, *}$; and \\ Yinghong Wang, $\mathrm{MD}, \mathrm{PhD}^{3, *}$
}

\begin{abstract}
Background: Immune checkpoint inhibitor (ICI) therapy predisposes patients to immune-related adverse events (irAEs). Data are limited regarding the incidence, management, and outcomes of one such irAE: mucositis. In this study, we evaluated the clinical characteristics, disease course, treatment, and outcomes of $\mathrm{ICl}$-mediated mucositis. Methods: This was a retrospective, single-center study of patients who received $\mathrm{ICl}$ therapy and developed oral mucositis at The University of Texas MD Anderson Cancer Center from January 2009 to September 2019. Inclusion criteria included age $\geq 18$ years, a diagnosis of oral mucositis and/or stomatitis based on ICD-9 and ICD-10 codes, and therapy using CTLA-4 or PD-1/L1 inhibitors alone or combined with other agents. Results: We identified 152 patients with a mean age of 60 years, $51 \%$ of whom were men. Of the sample patients, $73 \%$ had stage IV cancer, with melanoma the most common (28\%). Median time from ICl initiation to mucositis was 91 days. The most common clinical presentation of mucositis was odynophagia and/or oral pain (89\%), 91\% developed CTCAE grade 1-2 mucositis, and $78 \%$ received anti-PD-1/L1 monotherapy. Compared with anti-PD-1/L1-based therapy, anti-CTLA-4-based therapy was more frequently associated with earlier onset of mucositis (73 vs 96 days; $P=.077)$ and a lower rate of symptom resolution $(76 \%$ vs $92 \%$; $P=.029) ; 24 \%$ of patients required immunosuppressive therapy, which was associated with longer symptom duration (84 vs 34 days; $P=.002)$ and higher mucositis recurrence rate (61\% vs $32 \% ; P=.006)$. $\mathrm{ICl}$ interruption was associated with worse survival $(P=.037)$. Mucositis recurrence, immunosuppressant use, and presence of other irAEs did not affect survival. Conclusions: For ICl-mediated mucositis, a diagnosis of exclusion has not been well recognized and is understudied. Although the clinical symptoms of mucositis are mostly mild, approximately $25 \%$ of patients require immunosuppression. Mucositis recurrence can occur in approximately $39 \%$ patients. Our results showed that $\mathrm{ICl}$ interruption compromises overall survival.
\end{abstract}

J Natl Compr Canc Netw 2021;19(12):1415-1424 doi: 10.6004/jnccn.2020.7697

See JNCCN.org for supplemental online content.

\section{Background}

Immune checkpoint inhibitors (ICIs) are immunotherapeutic agents that inhibit checkpoint ligands and receptors that serve as downregulators of the adaptive and innate immune system, stimulating an immune response against cancer cells. Since their development, such as inhibitors of CTLA-4, PD-1, and PD-L1, ICIs have been found to be effective treatment options for various solid tumors, including melanoma; lung, genitourinary, colorectal, and head and neck malignancies; and leukemias. ${ }^{1-5}$

Although ICIs have changed the landscape of cancer therapy, their enhancement of nonspecific immune responses can lead to immune-related adverse events (irAEs) of any organ system. These irAEs are observed in the skin, gastrointestinal tract, liver, lungs, and other systems. ${ }^{4,5-8}$ irAEs occur at any time during ICI treatment, range from mild symptoms to severe life-threatening or fatal events, and may require management using immunomodulatory agents and discontinuation of ICI therapy., ${ }^{5,69}$

Gastrointestinal irAEs frequently involve the colon, liver, and stomach and present with nausea, vomiting, diarrhea, rectal bleeding, or abdominal pain. ${ }^{9-14}$ Rare gastrointestinal irAEs have been reported, such as

\footnotetext{
${ }^{1}$ Department of Internal Medicine, Baylor College of Medicine, Houston, Texas; ${ }^{2}$ Department of Internal Medicine, The University of Texas Health Science Center at Houston, Houston, Texas; ${ }^{3}$ Department of Gastroenterology, Hepatology, and Nutrition, The University of Texas MD Anderson Cancer Center, Houston, Texas; ${ }^{4}$ Department of Oncology, The Second Xiangya Hospital of Central South University, Changsha, Hunan, China; ${ }^{5}$ Department of Hepatobiliary and Pancreatic Surgery, Zhongnan Hospital of Wuhan University, Wuhan, China; ${ }^{6}$ Department of Medicine, University of Washington, Fred Hutchinson Cancer Research Center/Seattle Cancer Care Alliance, Seattle, Washington; and ${ }^{7}$ Department of Thoracic/Head \& Neck Medical Oncology, and ${ }^{8}$ Department of Melanoma Medical Oncology, The University of Texas MD Anderson Cancer Center, Houston, Texas.

*These authors contributed equally and are co-senior authors.
} 
esophagitis, pancreatitis, and cholecystitis. ${ }^{15-17}$ Furthermore, investigators have described adverse events in the oral cavity, including xerostomia, dysgeusia, lichenoid reactions, mucositis, and stomatitis. ${ }^{18-28}$

The clinical presentation of ICI-mediated mucositis (IMM) can range from mild to severe, with onset ranging from several weeks to months after treatment initiation. ${ }^{18-23}$ The literature contains several reports of severe mucositis induced by pembrolizumab, an anti-PD-1 agent, ${ }^{24-27}$ with symptom onset occurring up to 1 year after initiation and mucositis lasting several months after discontinuation. Physical and endoscopic findings showed mucosal erythema, edema, and ulceration with odynophagia and dysphagia symptoms, resulting in limited food intake, weight loss, and hospitalization. Successful management was accomplished via discontinuation of ICI and immunosuppression using steroids.

The pathogenesis of irAEs has yet to be fully elucidated, and their occurrence can impact quality of life and the ICI therapy course and lead to morbidity and mortality. Given the limited data on IMM, we evaluated the clinical characteristics, disease course, treatment, and outcome of IMM in patients at a tertiary cancer center.

\section{Methods}

\section{Study Design and Population}

This was a retrospective, descriptive, single-center study of adult patients at The University of Texas MD Anderson Cancer Center who received ICI therapy and experienced oral mucositis from January 2009 through September 2019. Inclusion criteria included age $\geq 18$ years, a diagnosis of oral mucositis and/or stomatitis based on ICD-9 and ICD-10 codes, and therapy using CTLA- 4 or PD-1/L1 inhibitors alone or combined with other agents. Patients who received a combination of ICI and non-ICI chemotherapy were included. The event time window for mucositis/stomatitis was from administration of the first ICI dose to 6 months after the last ICI dose. Exclusion criteria included (1) radiation therapy to the head, neck, or chest within 3 months of mucositis/stomatitis onset; (2) stem cell or bone marrow transplantation with gastrointestinal graft-versus-host disease; (3) confirmed upper gastrointestinal viral, fungal, or bacterial infection; (4) cancer in the oral cavity; and (5) mucositis resulting from other non-ICI cancer treatment. In patients in whom the etiology of mucositis was ambiguous, attribution to ICI therapy was made based on typical timing of disease onset from initiation of ICI therapy.

\section{Clinical Data}

Patient demographics, medical and oncologic history, cancer treatment regimens, and irAEs were collected. Demographics included age, sex, and ethnicity. Comorbidities evaluated included hypertension, coronary artery disease, congestive heart failure, atrial fibrillation, cerebral vascular disease, diabetes mellitus, chronic obstructive pulmonary disease, asthma, HIV infection, and thyroid disease. Information collected regarding presumed risk factors for mucositis included smoking history, use of nonsteroidal anti-inflammatory agents, other concurrent chemotherapy using ICIs, and premucositis use of immunosuppressants. Oncologic data analyzed included cancer type and stage, cancer treatment agents (ICI and non-ICI chemotherapy), and irAEs. ICIs administered included ipilimumab, nivolumab, pembrolizumab, atezolizumab, durvalumab, avelumab, cemiplimab, and tremelimumab. ICI regimens were categorized as single-agent or combined regimens. The duration of each ICI regimen before onset of mucositis was recorded. Information regarding mucositis and other irAEs within the same study window and their medical treatments was also documented.

\section{Mucositis Data}

Mucositis events were screened for time of onset, duration, severity, treatment, complications, recurrence, and need to hold or discontinue the ICI regimen. Mucositis severity was graded based on version 5 of the NCI's CTCAE. ${ }^{28}$ Signs of mucositis identified in history and physical examination were recorded as ulcers/aphthae or nonulcer inflammation. Symptoms included oral pain, odynophagia, dysphagia, nausea or vomiting, and bleeding. Treatment of mucositis was categorized as nonimmunosuppressive (proton pump inhibitors [PPIs], H2 blockers, sucralfate, or topical anesthetics) or immunosuppressive, including topical agents (eg, liquid dexamethasone, fluocinonide ointment) and systemic agents (intravenous methylprednisolone, oral dexamethasone, and oral prednisone). Duration of systemic steroid use was defined as the time from initiation to either cessation of treatment or return to the baseline maintenance dose. Clinical outcomes included requirement of hospitalization, duration of hospital stay, requirement of additional nutrition support, clinical resolution and recurrence of mucositis, and overall survival (OS) after mucositis onset.

\section{Statistical Analysis}

Statistical analysis was performed using RStudio version 1.0.136 (R Foundation for Statistical Computing) and SPSS Statistics, version 24.0 (IBM Corp). Categorical variables were summarized using frequencies and percentages. Continuous variables were summarized using means and standard deviations or medians and interquartile ranges (IQRs). A chi-square test was used to determine associations between categorical variables. The Mann-Whitney $U$ test was used to compare differences between continuous variables. Differences in OS durations between groups were analyzed using 


\section{Table 1. Baseline Clinical Characteristics}

\begin{tabular}{|c|c|}
\hline Characteristic & $n(\%)$ \\
\hline Total, N & 152 \\
\hline Mean age $[S D], y$ & 60.0 [13.6] \\
\hline Male sex & $77(51)$ \\
\hline \multicolumn{2}{|l|}{ Race } \\
\hline White & $109(72)$ \\
\hline Black & $5(3)$ \\
\hline Hispanic & $20(13)$ \\
\hline Other & $18(12)$ \\
\hline Comorbidities & $117(77)$ \\
\hline \multicolumn{2}{|l|}{ Baseline risk factors } \\
\hline NSAID use & $28(18)$ \\
\hline History of smoking & $72(47)$ \\
\hline Premucositis immunosuppressant use & $36(24)$ \\
\hline Other concurrent chemotherapy ${ }^{\mathrm{a}}$ with $\mathrm{ICls}$ & $87(57)$ \\
\hline \multicolumn{2}{|l|}{ Antacid medication } \\
\hline PPI & $48(32)$ \\
\hline $\mathrm{H} 2$ blocker & $20(13)$ \\
\hline \multicolumn{2}{|l|}{ Cancer type } \\
\hline Melanoma & $42(28)$ \\
\hline Lung or head and neck & $27(18)$ \\
\hline Genitourinary & $26(17)$ \\
\hline $\mathrm{Gl}$ & $16(11)$ \\
\hline Endocrine & $6(4)$ \\
\hline Other solid tumors & $13(9)$ \\
\hline Hematologic malignancies & $25(16)$ \\
\hline \multicolumn{2}{|l|}{ Cancer stage $^{\mathrm{b}}$} \\
\hline III & $13(9)$ \\
\hline IV & $111(73)$ \\
\hline
\end{tabular}

(continued)

the Kaplan-Meier method and a log-rank test. $P$ values up to .05 were considered significant.

\section{Results}

\section{Baseline Characteristics}

Of the 10,275 patients who received ICI therapy during the study period, 652 (6.3\%) developed mucositis, 500 of whom were excluded (supplemental eFigure 1, available with this article at JNCCN.org); 152 patients were included in the final analysis. Mean age at ICI initiation was 60 years, $51 \%$ were men, and $72 \%$ were White (Table 1 ). Concurrent chemotherapy in addition to ICIs (57\%) and history of smoking $(47 \%)$ were the most prevalent baseline risk factors, and the most common malignancies were melanoma (28\%), lung (10\%), head and neck (8\%), and hematologic (16\%). Most patients $(73 \%)$ had stage IV disease.

\begin{tabular}{|ll|}
\hline \multicolumn{2}{|l|}{ Table 1. Baseline Clinical Characteristics (cont.) } \\
\hline Characteristic & $\mathbf{n}(\%)$ \\
\hline $\begin{array}{l}\text { Median number of ICl therapy cycles before } \\
\text { mucositis diagnosis (IQR) }\end{array}$ & $3(1-6)$ \\
\hline ICI type & $33(22)$ \\
\hline CTLA-4 inhibitor ${ }^{c}$ & $119(78)$ \\
\hline PD-1/L1 inhibitor & \\
\hline \begin{tabular}{l} 
Other irAEs \\
\hline Gl toxicity
\end{tabular} & $7(5)$ \\
\hline Upper GI tract (esophagus-jejunum) & $18(12)$ \\
\hline Lower GI tract (ileum and colon) & $13(9)$ \\
\hline Liver & $9(6)$ \\
\hline Endocrine & $12(8)$ \\
\hline Lung & $41(27)$ \\
\hline Skin & $34(22)$ \\
\hline Hematologic & $18(12)$ \\
\hline Other &
\end{tabular}

Abbreviations: $\mathrm{Gl}$, gastrointestinal; $\mathrm{ICl}$, immune checkpoint inhibitor; $I Q R$, interquartile range; irAE, immune-related adverse event; NSAID, nonsteroidal anti-inflammatory drug; PPI, proton pump inhibitor.

${ }^{a}$ Classes of chemotherapy included antimetabolites, kinase inhibitors, alkylating agents, anthracyclines, MEK inhibitors, angiogenesis inhibitors, CCR4 inhibitors, and selective inhibitors of nuclear export.

${ }^{\mathrm{b}}$ For solid tumors, cancer stage was recorded based on the TNM Staging System. Stage was not recorded for hematologic cancers. Comorbidities were myocardial infarction/angina, coronary artery disease with percutaneous coronary intervention or coronary artery bypass graft, congestive heart failure, chronic obstructive pulmonary disease, HIV infection, hypocortisolism, atrial fibrillation, graft-versus-host disease, asthma, hypertension, diabetes mellitus, cerebral vascular disease, and thyroid disease.

${ }^{\mathrm{C}}$ Anti-CTLA-4 therapy included monotherapy and combination with anti-PD-1/L1 agents.

\section{Mucositis-Related Characteristics}

Among different ICI regimens, 119 patients (78\%) received PD-1/L1 monotherapy and 33 (22\%) received CTLA-4-based therapy. In the latter group, 16 received monotherapy and 17 received combination therapy (Table 2). Clinically, patients presented most commonly with odynophagia and/or oral pain $(89 \%)$ followed by nausea or vomiting $(20 \%)$. Most patients $(91 \%)$ had mild disease, with a peak CTCAE grade of 1 or 2. Median time of mucositis onset after ICI initiation was 91 days (IQR, 27-174 days), with a shorter duration in the anti-CTLA-4 versus the anti-PD-1/L1 group (73 vs 96 days, respectively; $P=.077$ ). Nearly all patients (97\%) presented with oral ulcers or aphthae (Figure 1), with a median duration of symptoms of 48 days (IQR, 19-99 days). Although most patients received supportive therapy, $36(24 \%)$ needed immunosuppressants (systemic or topical steroids), with a median duration of 51 days (IQR, 26-81 days). Of these patients, 22 received systemic steroids alone, 7 received topical steroids alone, and the remaining 7 


\begin{tabular}{|c|c|c|c|c|}
\hline Characteristic & $\begin{array}{l}\text { Overall } \\
\text { n (\%) }\end{array}$ & $\begin{array}{l}\text { Anti-CTLA-4 } \\
\text { n (\%) }\end{array}$ & $\begin{array}{l}\text { Anti-PD-1/L1 } \\
\mathrm{n}(\%)\end{array}$ & $P$ Value \\
\hline Total, $\mathrm{n}$ & 152 & 33 & 119 & \\
\hline Median duration from ICI therapy to mucositis onset (IQR), $d$ & $91(27-174)$ & $73(22-119)$ & $96(35-199)$ & .077 \\
\hline \multicolumn{5}{|l|}{ Clinical presentation } \\
\hline Odynophagia/Oral pain & $136(89)$ & $28(85)$ & $108(91)$ & .511 \\
\hline Nausea or vomiting & $31(20)$ & $7(21)$ & $24(20)$ & 1.000 \\
\hline Dysphagia & $15(10)$ & $1(3)$ & $14(12)$ & .247 \\
\hline Bleeding & $6(4)$ & $3(9)$ & $3(3)$ & .226 \\
\hline Peak CTCAE grade of mucositis & & & & .630 \\
\hline $1-2$ & $139(91)$ & $29(88)$ & $110(92)$ & \\
\hline $3-4$ & $13(9)$ & $4(12)$ & $9(8)$ & \\
\hline Physical examination finding & & & & 1.000 \\
\hline Ulcers/Aphthae & $148(97)$ & $32(97)$ & $116(97)$ & \\
\hline Nonulcer inflammation & $4(3)$ & $1(3)$ & $3(3)$ & \\
\hline Median duration of symptoms (IQR), $d ; n=133$ & 48 (19-99) & $35(21-77)$ & $56(18-112)$ & .400 \\
\hline Presence of other irAEs & $98(64)$ & $27(82)$ & $71(60)$ & .032 \\
\hline $\mathrm{ICl}$ therapy interrupted & $117(77)$ & $29(88)$ & $88(74)$ & .148 \\
\hline \multicolumn{5}{|l|}{ Mucositis treatment } \\
\hline \multicolumn{5}{|l|}{ Immunosuppressants ${ }^{\mathrm{b}}$} \\
\hline Systemic \pm topical & $29(19)$ & $6(18)$ & $23(19)$ & .690 \\
\hline Topical alone & $7(5)$ & $2(6)$ & $5(4)$ & 1.000 \\
\hline Supportive medication only ${ }^{c}$ & $99(65)$ & $21(64)$ & $78(66)$ & 1.000 \\
\hline No medical treatment & $17(11)$ & $5(15)$ & $12(10)$ & .610 \\
\hline Median duration of immunosuppressant use (IQR), d; $n=24$ & $51(26-81)$ & $39(30-57)$ & $60(21-85)$ & .830 \\
\hline \multicolumn{5}{|l|}{ Outcome } \\
\hline Hospitalization & $17(11)$ & $3(9)$ & $14(12)$ & .905 \\
\hline Median duration of hospitalization (IQR), d; $n=29$ & $10(6-20)$ & $18(7-24)$ & $9(6-15)$ & .240 \\
\hline Malnutrition requiring additional nutrition support (tube feeding or TPN) & $6(4)$ & $2(6)$ & $4(3)$ & .840 \\
\hline Clinical improvement/resolution & $134(88)$ & $25(76)$ & $109(92)$ & .029 \\
\hline Recurrence of mucositis & $52(34)$ & $11(33)$ & $41(34)$ & .716 \\
\hline Rehospitalization for recurrent mucositis & $2(1)$ & 0 & $2(2)$ & 1.000 \\
\hline Resumed $\mathrm{ICl}$ therapy; $\mathrm{n}=117$ & $25(21)$ & $5(17)$ & $20(23)$ & .716 \\
\hline All-cause mortality & $76(50)$ & $19(58)$ & $57(48)$ & .430 \\
\hline
\end{tabular}

Abbreviations: ICl, immune checkpoint inhibitor; IQR, interquartile range; irAE, immune-related adverse event; PPI, proton pump inhibitor; TPN, total parenteral nutrition.

${ }^{a}$ Anti-CTLA-4 therapy included monotherapy $(n=16)$ and combination with anti-PD-1/L1 agents $(n=17)$.

b22 patients used systemic immunosuppressants only, 7 used topical immunosuppressants only, and 7 used both topical and systemic immunosuppressants.

These agents included intravenous methylprednisolone, oral dexamethasone, oral prednisone, topical dexamethasone, and topical fluocinonide.

'Supportive medications included PPIs, H2 blockers, sucralfate, and viscous lidocaine.

received a combination. No patient received biologic therapy for mucositis.

Overall, $88 \%$ of patients had clinical improvement or resolution of mucositis: $92 \%$ in the anti-PD-1/L1 group versus $76 \%$ in the anti-CTLA-4 group $(P=.029)$. Seventeen patients (11\%) were hospitalized for a median stay of 10 days (IQR, 6-20 days). Of those hospitalized, $4(24 \%)$ had central line placement due to mucositis and 1 (6\%) had line-related bloodstream infection. Six patients $(4 \%)$ had mucositis compromising nutrition, necessitating support via tube feeding (2 using nasogastric feeding, 4 using percutaneous endoscopic gastrostomy tube) or total parenteral nutrition. Thirty-four percent of patients had recurrence of mucositis, leading to $1.3 \%$ of patients requiring rehospitalization. Among all patients, $64 \%$ had irAEs in 

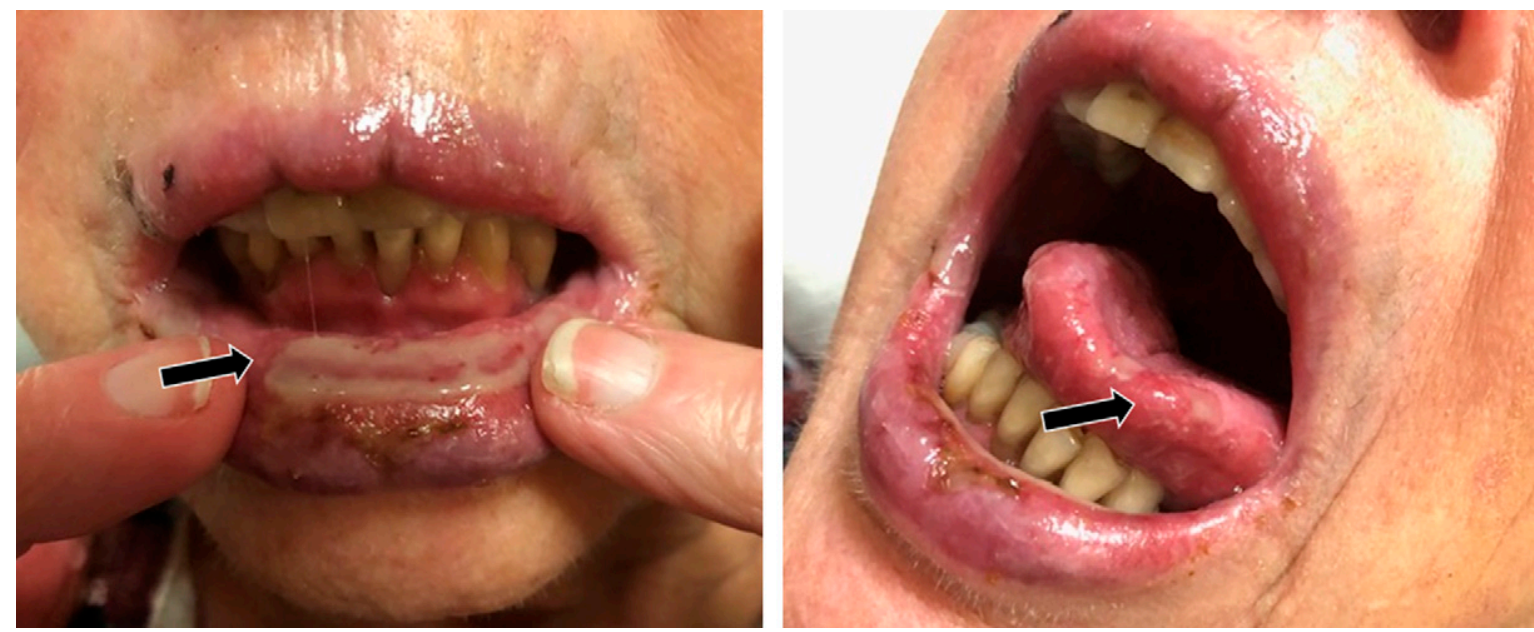

Figure 1. Oral mucositis.

(Courtesy of Andrea M. Perdue and John A. Thompson at the University of Washington, Seattle, Washington).

addition to mucositis, with a higher incidence in patients given CTLA-4-based regimens than in those with anti-PD-1/L1-based regimens $(82 \%$ vs $60 \%$; $P=.032$ ). Of these irAEs, $25 \%$ involved the gastrointestinal system, including gastritis and enterocolitis. ICI therapy was interrupted in $77 \%$ of patients because of irAEs. The all-cause mortality rate at the end of the study period was $50 \%$ (Table 2 ).

Immunosuppressive therapy was associated with a longer duration of mucositis-related symptoms compared with nonimmunosuppressive therapy (84 vs 34 days; $P=.002$ ) (Table 3 ). The other parameters were comparable in these 2 groups. In the immunosuppressive therapy group, patients who received topical therapy generally had milder symptoms compared with those who received systemic therapy, and all patients had resolution of their symptoms $(P>.05$; supplemental eTable 1).

We found that patients receiving anti-CTLA-4 therapy were more likely to have mucositis and other irAEs than were those receiving anti-PD-1/L1 therapy $(P=.032$; supplemental eTable 2). Basic risk factors at the time of mucositis diagnosis did not affect the severity or outcomes of mucositis.

\section{Recurrence of Mucositis}

Among 134 patients who achieved symptom improvement/resolution, 52 (39\%) had recurrence of mucositis (Table 4). Recurrence was associated with the presence of coexisting irAEs $(P=.038)$ and the requirement of immunosuppression for the initial mucositis event $(P=.006)$. Median duration of symptoms was longer in patients with recurrence versus without (56 vs 36 days; $P=.058$ ). Univariate logistic regression analysis showed that the following factors were significantly associated with mucositis recurrence: dysphagia symptoms (odds ratio $[\mathrm{OR}], 3.222 ; P=.047$ ), longer duration of symptoms (OR, 1.003; $P=.051$ ), and immunosuppressant use for the initial mucositis event (OR, 3.317; $P=.004$ ) (Table 5).

\section{Overall Survival}

Patients in whom ICI therapy was continued or resumed after interruption had significantly favorable OS $(P=.037$; Figure 2$)$. We observed a complete separation in the OS curves for patients with and without recurrent mucositis, although the difference was not significant (supplemental eFigure 2A). In addition, OS was not associated with immunosuppressant use or the presence of other irAEs along with mucositis $(P>.05)$ (supplemental eFigure 2B, C).

\section{Discussion}

ICI therapy can have a dramatic positive impact on OS in patients with advanced cancers. ${ }^{1-4}$ However, decreased immune regulation can involve a breakdown of tolerance to self-antigens, leading to the development of irAEs. ${ }^{5}$ Oral mucositis has not been well studied given the variations in its diagnosis, determination of causality, and mild presentation when compared with other irAEs. ${ }^{25}$ This study is the first focused analysis with the largest sample size to characterize the clinical presentations, response to therapy, and outcomes of ICI-related oral mucositis.

Our study showed that the median time to onset of mucositis was 3 months after ICI initiation, similar to ICI colitis. ${ }^{29}$ Patients receiving anti-CTLA-4-based therapy had earlier onset of mucositis, lower mucositis treatment response rates, and more irAEs than did those receiving anti-PD-1/L1 therapy, although the 


\begin{tabular}{|c|c|c|c|}
\hline Characteristic & $\begin{array}{l}\text { Immunosuppressive } \\
\text { Treatment } \\
\text { n (\%) }\end{array}$ & $\begin{array}{l}\text { Nonimmunosuppressive } \\
\text { Treatment } \\
\text { n (\%) }\end{array}$ & $P$ Value \\
\hline Total, $\mathrm{n}$ & $36^{\mathrm{a}}$ & 116 & \\
\hline $\begin{array}{l}\text { Median duration from } \mathrm{ICl} \text { therapy } \\
\text { to mucositis onset (IQR), d }\end{array}$ & $97(44-206)$ & $87(23-170)$ & .230 \\
\hline \multicolumn{4}{|l|}{ Clinical presentation } \\
\hline Odynophagia/Oral pain & $35(97)$ & $101(87)$ & .155 \\
\hline Nausea or vomiting & $6(17)$ & $25(22)$ & .690 \\
\hline Dysphagia & $5(14)$ & $10(9)$ & .545 \\
\hline Bleeding & 0 & $6(5)$ & .367 \\
\hline Peak grade of mucositis & & & .770 \\
\hline $1-2$ & $32(89)$ & 107 (92) & \\
\hline $3-4$ & $4(11)$ & $9(8)$ & \\
\hline Physical examination finding & & & 1.000 \\
\hline Ulcers/Aphthae & $35(97)$ & $113(97)$ & \\
\hline Nonulcer inflammation & $1(3)$ & $3(3)$ & \\
\hline Median duration of symptoms (IQR), d; $n=133$ & $84(46-120)$ & $34(14-84)$ & .002 \\
\hline Other coexisting irAEs within study window & $25(69)$ & $73(63)$ & .607 \\
\hline Gastrointestinal irAEs & $8(22)$ & $16(14)$ & .342 \\
\hline \multicolumn{4}{|l|}{ Outcome } \\
\hline Hospitalization & $6(17)$ & $11(9)$ & .370 \\
\hline Median hospital stay (IQR), d; $n=29$ & $8(4-16)$ & $11(7-21)$ & .350 \\
\hline $\begin{array}{l}\text { Malnutrition requiring additional nutrition } \\
\text { support (tube feeding or TPN) }\end{array}$ & 0 & $6(5)$ & .370 \\
\hline Clinical improvement/resolution & $33(92)$ & $101(87)$ & .652 \\
\hline Recurrence of mucositis; $n=134$ & $20(61)$ & $32(32)$ & .006 \\
\hline All-cause mortality & $14(39)$ & $62(53)$ & .180 \\
\hline
\end{tabular}

Abbreviations: ICl, immune checkpoint inhibitor; IQR, interquartile range; irAE, immune-related adverse event; TPN, total parenteral nutrition.

a22 patients used systemic immunosuppressants only, 7 used topical immunosuppressants only, and 7 used both topical and systemic immunosuppressants. These agents included intravenous methylprednisolone, oral dexamethasone, oral prednisone, topical dexamethasone, and topical fluocinonide.

peak CTCAE grade of symptoms was comparable. irAEs of greater toxicity are more frequently related to anti-CTLA-4 therapy, ${ }^{30-33}$ possibly because of the different mechanisms of action of these agents. Although PD-1/Ll inhibition restores immune function and decreases the regulation of specific $\mathrm{T}$ cells inactivated due to high levels of antigen exposure, CTLA- 4 inhibition leads to the activation and proliferation of a large number of effector $\mathrm{T}$ cells and decreases their regulation regardless of T-cell specificity, potentially leading to greater autoreactivity. ${ }^{34}$ Both mechanisms can lead to severe irAEs.

In our sample, most patients with mucositis had mild symptoms (CTCAE grade 1 or 2). Although $11 \%$ of patients did not receive treatment, the remainder needed supportive care $(65 \%)$ or immunosuppressive therapy (24\%). Management of mucositis was highly variable due to a lack of available guidelines. Most management strategies are extrapolated from guidelines for mucositis secondary to irradiation or treatment using other antineoplastic agents, ${ }^{35,36}$ although the pathophysiology differs starkly. Notably, regardless of the use of immunosuppressive therapy in patients with long symptom durations, we found negligible improvement in their clinical outcomes (eg, hospitalization, length of hospital stay, mucositis response rate) and a dramatically higher recurrence rate. These findings question the benefit of corticosteroids in the management and outcomes of mucositis and highlight potential complications. ${ }^{37}$ However, disease severity, timing of immunosuppressive therapy initiation, treatment duration, and concomitant medications and comorbidities can also confound the outcomes.

In our cohort, mucositis improved with supportive care in most patients. Sucralfate, an agent that creates a protective mucosal barrier, had mixed outcomes in 


\begin{tabular}{|c|c|c|c|}
\hline $\mathrm{ICl}$ type & & & .716 \\
\hline Anti-CTLA-4 therapy & $11(21)$ & $14(17)$ & \\
\hline Odynophagia/Oral pain & $48(92)$ & $73(89)$ & .744 \\
\hline Nausea or vomiting & $5(10)$ & $23(28)$ & .075 \\
\hline Dysphagia & $9(17)$ & $5(6)$ & .019 \\
\hline Bleeding & $1(2)$ & $4(5)$ & .681 \\
\hline Other coexisting irAEs & $38(73)$ & $44(54)$ & .038 \\
\hline Gastrointestinal irAEs & $11(21)$ & $10(12)$ & .252 \\
\hline Median duration of symptoms (IQR), $d ; n=51$ and $n=75$ & $56(21-181)$ & $36(15-93)$ & .058 \\
\hline Hospitalization & $3(6)$ & $8(10)$ & .620 \\
\hline \multicolumn{4}{|l|}{ Mucositis treatment } \\
\hline Immunosuppressants & $20(38)$ & $13(16)$ & .006 \\
\hline $\begin{array}{l}\text { Duration from onset of symptoms } \\
\text { to immunosuppressant use (IQR), } d ; n=36\end{array}$ & $10(0-43)$ & $7.5(0-29)$ & .728 \\
\hline Duration of immunosuppressant use (IQR), $d ; n=36$ & $38(23-63)$ & $67(34-88)$ & .290 \\
\hline $\mathrm{ICl}$ therapy resumption & $13(25)$ & $12(15)$ & .203 \\
\hline
\end{tabular}

Abbreviations: $I C l$, immune checkpoint inhibitor; IQR, interquartile range; irAE, immune-related adverse event.

mucositis treatment, ${ }^{38}$ although a few trials have shown improvement of radiation- and 5-FU-induced oral mucositis. ${ }^{39,40}$ Small case series have shown improvement of radiation-induced mucositis using PPIs, although they seem to be helpful only in patients with severe reflux symptoms. ${ }^{41}$ Although authors have cautioned against the use of PPIs without clear indication, ${ }^{42}$ largely due to the risk of hypersensitivity and cutaneous reactions, ${ }^{43-46}$ our findings illustrate the benefit of their use as first-line therapy for mild mucositis.

Topical immunosuppressive agents can serve as alternatives to systemic corticosteroids given their highly favorable adverse effect profiles. In our cohort, we observed that patients who received topical steroid therapy alone had lower mucositis grades, complete symptom improvement, fewer hospitalizations, and shorter hospital stays than did those undergoing systemic immunosuppression. In previous case studies, the use of topical corticosteroids in the management of inflammatory mucositis in patients with graft-versus-host disease was promising. ${ }^{47-49}$ Bertelli et $\mathrm{al}^{49}$ described a patient with severe oral mucositis limiting nutritional intake whose condition was completely resolved using systemic methylprednisolone followed by oral viscous budesonide. Our findings suggest that topical immunosuppressants can be beneficial in the management of IMM, which should be further explored.

Our findings illustrate the critical need for a standardized approach to managing IMM. Topical agents should be considered before systemic therapy. Elad et al ${ }^{50}$ reported the efficacy of topical immunomodulators (azathioprine, benzydamine, and tetracyclines) in managing IMM refractory to topical corticosteroids. In addition, high-risk patients who would benefit from maintenance therapy to prevent recurrence of IMM should be identified. If patients are refractory to steroid treatment, then biologics may be considered as a potential salvage option. For instance, Horisberger et $\mathrm{al}^{51}$ reported success in treating refractory mucositis and esophageal stenosis using anti-PD-1 therapy and tocilizumab, an IL-6 blocker. Other studies have shown promising outcomes in patients with immune-mediated colitis treated using infliximab or vedolizumab as adjunct or salvage therapy, with $70 \%$ to $85 \%$ clinical improvement, and a much lower recurrence rate with 


\begin{tabular}{|lll|}
\hline $\begin{array}{l}\text { Table 5. Univariate Logistic Regression Analysis } \\
\text { for Recurrence of Mucositis }\end{array}$ & P Value \\
\hline Characteristic & OR (95\% CI) & A \\
\hline Age & $0.997(0.971-1.023)$ & .804 \\
\hline Male sex & $0.952(0.474-1.912)$ & .891 \\
\hline Basic risk factors & $0.746(0.318-1.778)$ & .502 \\
\hline NSAID use & $1.198(0.492-2.848)$ & .683 \\
\hline History of smoking & $0.645(0.317-1.298)$ & .222 \\
\hline \multicolumn{1}{|c|}{ Concurrent chemotherapy } & $0.765(0.379-1.541)$ & .453 \\
\hline Concurrent GI toxic effects & $1.931(0.753-5.018)$ & .169 \\
\hline Anti-CTLA-4 regimen ${ }^{2}$ & $1.303(0.532-3.136)$ & .555 \\
\hline Odynophagia/Oral pain & $1.480(0.454-5.708)$ & .533 \\
\hline Nausea or vomiting & $0.273(0.086-0.721)$ & .014 \\
\hline Dysphagia & $3.222(1.045-11.065)$ & .047 \\
\hline Duration of symptoms & $1.003(1.000-1.006)$ & .051 \\
\hline Continued ICl therapy & $1.944(0.806-4.731)$ & .137 \\
\hline $\begin{array}{l}\text { Duration from ICI therapy } \\
\text { to mucositis onset }\end{array}$ & $1.000(0.999-1.002)$ & .736 \\
\hline Required immunosuppressive therapy & $3.317(1.485-7.645)$ & .004 \\
\hline $\begin{array}{l}\text { Duration of immunosuppressive } \\
\text { therapy }\end{array}$ & $1.000(0.981-1.024)$ & .530 \\
\hline Hospitalization & $0.566(0.120-2.066)$ & .418 \\
\hline
\end{tabular}

Abbreviations: $\mathrm{Gl}$, gastrointestinal; ICl, immune checkpoint inhibitor; NSAID, nonsteroidal anti-inflammatory drug; OR, odds ratio.

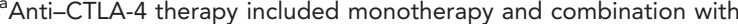
anti-PD-1/L1 agents.

vedolizumab maintenance therapy among patients at high risk for recurrence of immune-mediated colitis. ${ }^{52-55}$ Unfortunately, evidence regarding the efficacy of biologic therapy for upper gastrointestinal toxicities is limited.

In addition to medical options, oral microbial derangements were recently found to be associated with recurrent aphthous stomatitis and ulceration. ${ }^{56-58}$ Wang et al ${ }^{59}$ showed that the reconstitution of the gut microbiome via fecal microbiota transplantation in patients with immunemediated colitis led to the resolution of inflammation and the upregulation of colonic regulatory $\mathrm{T}$ lymphocytes. We speculated that oral microbiome modification may provide a similar benefit to mucositis and could be explored further as a novel therapeutic avenue.

As shown previously, irAEs are generally associated with improved OS, progression-free survival, and ICI efficacy in patients with advanced cancer. ${ }^{11,60-65}$ Severe forms of irAEs-higher CTCAE grade, more organ involvement, and irAE-related complications-have been associated with longer OS. ${ }^{17,37,65}$ An irAE may recur despite resolution of the initial event and ICI discontinuation, as reported previously. ${ }^{51,66-68}$ A severe, refractory irAE prompts cessation of ICI therapy for the underlying malignancy in view of poor quality of life, which is likely

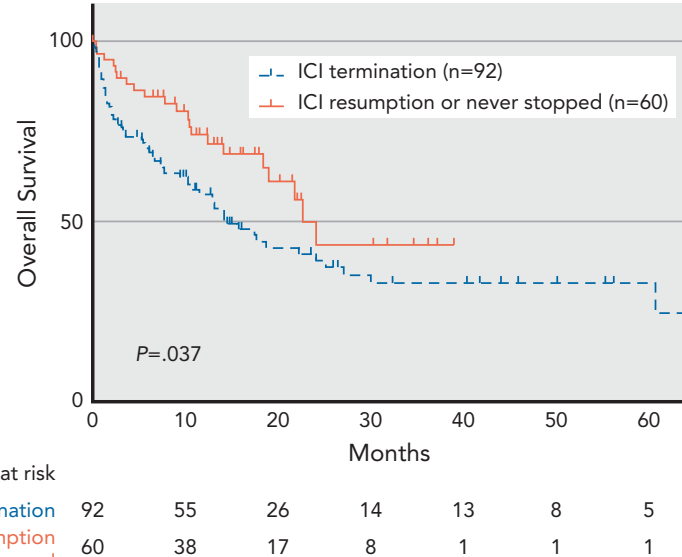

Figure 2. Overall survival based on $\mathrm{ICl}$ treatment status. Abbreviation: $\mathrm{ICl}$, immune checkpoint inhibitor.

reflective of the long-lasting systemic effect of ICIs and lingering toxicities that may be associated with favorable oncologic outcomes. This is supported by previous studies of other irAEs ${ }^{16,17}$ and suggested by the superior OS in patients with ICI continuation in this study. An OS benefit was not manifested in our patients with recurrent mucositis, perhaps because of the small sample size and other confounders. Overall, these findings raise the question of how to optimize ICI treatment duration while effectively managing irAEs.

Our study has some limitations. First, data collection and analysis were limited by the retrospective, singlecenter design and small sample size. Second, the true incidence and severity of mucositis may have been underrepresented with mild or subclinical cases. The CTCAE grading system, although standardized, relies purely on self-reported documentation, with wide variation. Third, diagnosis, monitoring, and management of mucositis were performed at the discretion of the provider and bore no standardized management approach. Patients who received treatment for coexisting irAEs may have confounded outcomes of mucositis. Finally, we did not perform landmark analysis given neglectable immortal time and did not conduct subgroup OS analysis for different types of malignancy because of the small sample size. However, despite these limitations, we present new, important data that generate hypotheses in a clinically relevant setting and that support further investigation of this topic.

\section{Conclusions}

IMM is an underrecognized irAE in patients receiving ICI therapy, and is often mild and effectively managed using supportive medications. Rarely, patients with severe IMM require hospitalization, immunosuppression, nutrition support, and disruption of ICI treatment. Our findings 
showed that up to $39 \%$ of patients may experience recurrence, which is associated with longer duration of symptoms and immunosuppressive therapy for the initial episode, and that ICI interruption due to irAEs shortens OS. Further studies are warranted to identify risk factors associated with refractory mucositis, clarify the role and logistics of immunosuppressive therapy, and develop standardized diagnostic and management strategies to optimize the clinical benefit of ICI therapy in patients with mucositis.

\section{Acknowledgments}

Editing of this article was provided by Scientific Publications, Research Medical Library at The University of Texas MD Anderson Cancer Center. Ethics approval for this study was granted by the Institutional Review Board of The University of Texas MD Anderson Cancer Center (PA18-0472). Patient consent was waived for this study. The datasets used and analyzed in this study are available from the corresponding author upon reasonable request.
Submitted August 18, 2020; final revision received October 21, 2020; accepted for publication December 7, 2020.

Published online August 4, 2021

Author contributions: Senior researchers: Thomas, Wang. Study concept and design: Thomas, Wang. Data collection: Jacob, Dutra, GarciaRodriguez, Panneerselvam, Abraham. Interpretation of results: Thomas, Wang. Formal analysis: Jacob, Dutra. Supervision: Thomas, Wang Manuscript-writing: Jacob, Dutra, Thomas, Wang. Manuscript-review and editing: Zou, Ma, Grivas, Thompson, Altan, Glitza Oliva, Zhang. Approval of final manuscript: All authors.

Disclosures: Dr. Grivas has disclosed serving as a consultant for AstraZeneca, Bayer, Biocept, Bristol Myers Squibb, Clovis Oncology Dyania Health, Driver, EMD Serono, Exelixis, Foundation Medicine, Genentech/Roche, Genzyme, GlaxoSmithKline, Heron Therapeutics, Immunomedics, Janssen, Merck, Mirati Therapeutics, Pfizer, Seattle Genetics, and QED Therapeutics; participating in an educational program for Bristol Myers Squibb; and receiving institutional research funding from AstraZeneca, Bavarian Nordic, Bayer, Bristol Myers Squibb, Clovis Oncology, Debiopharm, Genentech/Roche, GlaxoSmithKline,

Immunomedics, Merck, Mirati Therapeutics, Oncogenex, Pfizer, and QED Therapeutics. Dr. Wang has disclosed serving as a consultant for Tillotts Pharma. The remaining authors have disclosed that they have not received any financial consideration from any person or organization to support the preparation, analysis, results, or discussion of this article.

Correspondence: Yinghong Wang, MD, PhD, Department of Gastroenterology, Hepatology, and Nutrition, The University of Texas MD Anderson Cancer Center, Unit 1466, 1515 Holcombe Boulevard, Houston, TX 77030. Email: ywang59@mdanderson.org

\section{References}

1. Topalian SL, Drake CG, Pardoll DM. Immune checkpoint blockade: a common denominator approach to cancer therapy. Cancer Cell 2015;27: 450-461.

2. Hoos A. Development of immuno-oncology drugs-from CTLA4 to PD1 to the next generations. Nat Rev Drug Discov 2016;15:235-247.

3. Hodi FS, O'Day SJ, McDermott DF, et al. Improved survival with ipilimumab in patients with metastatic melanoma. N Engl J Med 2010;363: 711-723.

4. Pardoll DM. The blockade of immune checkpoints in cancer immunotherapy. Nat Rev Cancer 2012;12:252-264.

5. Postow MA, Sidlow R, Hellmann MD. Immune-related adverse events associated with immune checkpoint blockade. N Engl J Med 2018; 378:158-168.

6. Puzanov I, Diab A, Abdallah K, et al. Managing toxicities associated with immune checkpoint inhibitors: consensus recommendations from the Society for Immunotherapy of Cancer (SITC) Toxicity Management Working Group. J Immunother Cancer 2017;5:95.

7. Topalian SL, Hodi FS, Brahmer JR, et al. Safety, activity, and immune correlates of anti-PD-1 antibody in cancer. N Engl J Med 2012;366:2443-2454.

8. Weber JS, Yang JC, Atkins MB, et al. Toxicities of immunotherapy for the practitioner. J Clin Oncol 2015;33:2092-2099.

9. Kumar V, Chaudhary N, Garg M, et al. Current diagnosis and management of immune related adverse events (irAEs) induced by immune checkpoint inhibitor therapy. Front Pharmacol 2017:8:49.

10. Cramer P, Bresalier RS. Gastrointestinal and hepatic complications of immune checkpoint inhibitors. Curr Gastroenterol Rep 2017;19:3.

11. Wang $Y$, Abu-Sbeih $H$, Mao E, et al. Immune-checkpoint inhibitorinduced diarrhea and colitis in patients with advanced malignancies: retrospective review at MD Anderson. J Immunother Cancer 2018;6:37.

12. Karamchandani DM, Chetty R. Immune checkpoint inhibitor-induced gastrointestinal and hepatic injury: pathologists' perspective. J Clin Pathol 2018;71:665-671.

13. Reddy HG, Schneider BJ, Tai AW. Immune checkpoint inhibitorassociated colitis and hepatitis. Clin Transl Gastroenterol 2018;9:180

14. Tang $\mathrm{T}$, Abu-Sbeih $\mathrm{H}$, Luo W, et al. Upper gastrointestinal symptoms and associated endoscopic and histological features in patients receiving immune checkpoint inhibitors. Scand J Gastroenterol 2019;54:538-545.

15. Boike J, Dejulio T. Severe esophagitis and gastritis from nivolumab therapy. ACG Case Rep J 2017;4:e57.
16. Abu-Sbeih H, Tran CN, Ge PS, et al. Case series of cancer patients who developed cholecystitis related to immune checkpoint inhibitor treatment. J Immunother Cancer 2019;7:118.

17. Abu-Sbeih H, Tang T, Lu Y, et al. Clinical characteristics and outcomes of immune checkpoint inhibitor-induced pancreatic injury. J Immunother Cancer 2019;7:31

18. Ferris RL, Blumenschein $\mathrm{G} \mathrm{Jr}$, Fayette J, et al. Nivolumab for recurrent squamous-cell carcinoma of the head and neck. N Engl J Med 2016;375: 1856-1867.

19. McDermott DF, Sosman JA, Sznol M, et al. Atezolizumab, an antiprogrammed death-ligand 1 antibody, in metastatic renal cell carcinoma: long-term safety, clinical activity, and immune correlates from a phase la study. J Clin Oncol 2016;34:833-842.

20. Mok TSK, Wu YL, Kudaba I, et al. Pembrolizumab versus chemotherapy for previously untreated, PD-L1-expressing, locally advanced or metastatic non-small-cell lung cancer (KEYNOTE-042): a randomised, openlabel, controlled, phase 3 trial. Lancet 2019;393:1819-1830.

21. Motzer RJ, Escudier B, McDermott DF, et al. Nivolumab versus everolimus in advanced renal-cell carcinoma. N Engl J Med 2015;373: 1803-1813.

22. Freeman-Keller M, Kim $\mathrm{Y}, \mathrm{Cronin} \mathrm{H}$, et al. Nivolumab in resected and unresectable metastatic melanoma: characteristics of immune-related adverse events and association with outcomes. Clin Cancer Res 2016;22:886-894.

23. Fukui T, Okuma Y, Nakahara $Y$, et al. Activity of nivolumab and utility of neutrophil-to-lymphocyte ratio as a predictive biomarker for advanced non-small-cell lung cancer: a prospective observational study. Clin Lung Cancer 2019;20:208-214.e2.

24. Pelster MS, Mott F, Lewin J. Pembrolizumab-induced mucositis in a patient with recurrent hypopharynx squamous cell cancer. Laryngoscope 2020;130:E140-143.

25. Acero Brand FZ, Suter N, Adam JP, et al. Severe immune mucositis and esophagitis in metastatic squamous carcinoma of the larynx associated with pembrolizumab. J Immunother Cancer 2018;6:22

26. Zander T, Aebi S, Rast AC, et al. Response to pembrolizumab in a patient with relapsing thymoma. J Thorac Oncol 2016;11:e147-149.

27. Lederhandler $\mathrm{MH}, \mathrm{Ho} \mathrm{A}$, Brinster $\mathrm{N}$, et al. Severe oral mucositis: a rare adverse event of pembrolizumab. J Drugs Dermatol 2018;17:807-809.

28. National Cancer Institute. Common Terminology Criteria for Adverse Events (CTCAE) v5.0. Cancer Therapy Evaluation Program. Accessed December 14, 2020. Available at: https://ctep.cancer.gov/protocoldevelopment/electronic_applications/ctc.htm\#ctc_50 
29. Weber JS, Dummer R, de Pril V, et al. Patterns of onset and resolution of immune-related adverse events of special interest with ipilimumab: detailed safety analysis from a phase 3 trial in patients with advanced melanoma. Cancer 2013;119:1675-1682.

30. Michot JM, Bigenwald C, Champiat S, et al. Immune-related adverse events with immune checkpoint blockade: a comprehensive review. Eur J Cancer 2016;54:139-148.

31. Beck KE, Blansfield JA, Tran KQ, et al. Enterocolitis in patients with cancer after antibody blockade of cytotoxic T-lymphocyte-associated antigen 4. J Clin Oncol 2006;24:2283-2289.

32. Bertrand $A$, Kostine $M$, Barnetche $T$, et al. Immune related adverse events associated with anti-CTLA-4 antibodies: systematic review and meta-analysis. BMC Med 2015;13:211

33. Wang DY, Kim DW, Shah NJ, et al. Clinical presentation of immunerelated colitis associated with PD-1 inhibitor monotherapy (MONO) and combination PD-1/CTLA-4 inhibitors (COMBO) in melanoma [abstract]. J Clin Oncol 2017;35(Suppl):Abstract 9566.

34. Buchbinder El, Desai A. CTLA-4 and PD-1 pathways: similarities, differences, and implications of their inhibition. Am J Clin Onco 2016;39:98-106.

35. Peterson DE, Boers-Doets CB, Bensadoun RJ, et al. Management of oral and gastrointestinal mucosal injury: ESMO Clinical Practice Guidelines for diagnosis, treatment, and follow-up. Ann Oncol 2015;26(Supp 5):v139-151

36. Hong CHL, Gueiros LA, Fulton JS, et al. Systematic review of basic ora care for the management of oral mucositis in cancer patients and clinical practice guidelines. Support Care Cancer 2019;27:3949-3967.

37. Abu-Sbeih $H$, Tang $T$, Ali $F$, et al. The impact of immune checkpoint inhibitor-related adverse events and their immunosuppressive treatment on patients' outcomes. J Immunother Precis Oncol 2018;1:7-18.

38. Nottage M, McLachlan SA, Brittain MA, et al. Sucralfate mouthwash for prevention and treatment of 5-fluorouracil-induced mucositis: a randomized, placebo-controlled trial. Support Care Cancer 2003;11:41-47.

39. Cengiz M, Ozyar E, Oztürk D, et al. Sucralfate in the prevention of radiation-induced oral mucositis. J Clin Gastroenterol 1999;28:40-43.

40. Ala S, Saeedi M, Janbabai G, et al. Efficacy of sucralfate mouth wash in prevention of 5-fluorouracil induced oral mucositis: a prospective, randomized, double-blind, controlled trial. Nutr Cancer 2016;68:456-463.

41. Eguchi K, Suzuki M, Ida S, et al. Successful treatment of radiationinduced mucositis with proton pump inhibitor administration: a report of two laryngeal cancer cases. Auris Nasus Larynx 2017;44:122-125.

42. Leitinger $E$, Hui L, Grigg A. Is there a role for proton pump inhibitor prophylaxis in haematology patients? Intern Med J 2019;49:694-701.

43. Chang YS. Hypersensitivity reactions to proton pump inhibitors. Curr Opin Allergy Clin Immunol 2012;12:348-353.

44. Frey N, Bodmer M, Bircher A, et al. Stevens-Johnson syndrome and toxic epidermal necrolysis in association with commonly prescribed drugs in outpatient care other than anti-epileptic drugs and antibiotics: a population-based case-control study. Drug Saf 2019;42:55-66.

45. Chularojanamontri L, Jiamton S, Manapajon A, et al. Cutaneous reactions to proton pump inhibitors: a case-control study. J Drugs Dermatol 2012;11:e43-47.

46. Rodríguez-Martín S, Martín-Merino E, Lerma V, et al. Incidence of Stevens-Johnson syndrome/toxic epidermal necrolysis among new users of different individual drugs in a European population: a case-population study. Eur J Clin Pharmacol 2019;75:237-246.

47. Utsman RA, Epstein JB, Elad S. Budesonide for local therapy of complex oral mucosal immune-mediated inflammatory diseases: case reports. Oral Surg Oral Med Oral Pathol Oral Radiol Endod 2008;106:e11-17.

48. Noce CW, Gomes A, Shcaira V, et al. Randomized double-blind clinical trial comparing clobetasol and dexamethasone for the topical treatment of symptomatic oral chronic graft-versus-host disease. Biol Blood Marrow Transplant 2014;20:1163-1168.
49. Bertelli L, Di Nardo G, Zama D, et al. A new formulation of an old drug: a potential new therapy in the management of oral cGvHD. J Pediatr Hematol Oncol 2016;38:e295-297.

50. Elad S, Epstein JB, von Bültzingslöwen I, et al. Topical immunomodulators for management of oral mucosal conditions, a systematic review; part II: miscellaneous agents. Expert Opin Emerg Drugs 2011:16:183-202.

51. Horisberger A, La Rosa S, Zurcher JP, et al. A severe case of refractory esophageal stenosis induced by nivolumab and responding to tocilizumab therapy. J Immunother Cancer 2018;6:156.

52. Johnson $\mathrm{DH}$, Zobniw $\mathrm{CM}$, Trinh $\mathrm{VA}$, et al. Infliximab associated with faster symptom resolution compared with corticosteroids alone for the management of immune-related enterocolitis. J Immunother Cancer 2018;6:103.

53. Abu-Sbeih H, Ali FS, Wang X, et al. Early introduction of selective immunosuppressive therapy associated with favorable clinical outcomes in patients with immune checkpoint inhibitor-induced colitis. J Immunother Cancer 2019;7:93

54. Bergqvist V, Hertervig E, Gedeon P, et al. Vedolizumab treatment for immune checkpoint inhibitor-induced enterocolitis. Cancer Immunol Immunother 2017;66:581-592

55. Abu-Sbeih H, Ali FS, Alsaadi D, et al. Outcomes of vedolizumab therapy in patients with immune checkpoint inhibitor-induced colitis: a multicenter study. J Immunother Cancer 2018;6:142.

56. Stehlikova Z, Tlaskal V, Galanova N, et al. Oral microbiota composition and antimicrobial antibody response in patients with recurrent aphthous stomatitis. Microorganisms 2019;7:636

57. Hijazi K, Lowe T, Meharg C, et al. Mucosal microbiome in patients with recurrent aphthous stomatitis. J Dent Res 2015;94(3 Suppl):87S-94S.

58. Saikaly SK, Saikaly TS, Saikaly LE. Recurrent aphthous ulceration: a review of potential causes and novel treatments. J Dermatolog Treat 2018;29:542-552.

59. Wang Y, Wiesnoski DH, Helmink BA, et al. Fecal microbiota transplantation for refractory immune checkpoint inhibitor-associated colitis. Nat Med 2018;24:1804-1808.

60. Teulings HE, Limpens J, Jansen SN, et al. Vitiligo-like depigmentation in patients with stage III-IV melanoma receiving immunotherapy and its association with survival: a systematic review and meta-analysis. J Clin Oncol 2015;33:773-781.

61. Hua C, Boussemart L, Mateus $C$, et al. Association of vitiligo with tumor response in patients with metastatic melanoma treated with pembrolizumab. JAMA Dermatol 2016;152:45-51.

62. Nakamura $Y$, Tanaka $R$, Asami $Y$, et al. Correlation between vitiligo occurrence and clinical benefit in advanced melanoma patients treated with nivolumab: a multi-institutional retrospective study. J Dermato 2017:44:117-122.

63. Abu-Sbeih H, Ali FS, Qiao W, et al. Immune checkpoint inhibitor-induced colitis as a predictor of survival in metastatic melanoma. Cancer Immuno Immunother 2019;68:553-561.

64. Haratani $\mathrm{K}$, Hayashi $\mathrm{H}, \mathrm{Chiba} \mathrm{Y}$, et al. Association of immune-related adverse events with nivolumab efficacy in non-small cell lung cancer. JAMA Oncol 2018;4:374-378.

65. Wang $Y$, Abu-Sbeih $\mathrm{H}, \mathrm{Mao}$ E, et al. Endoscopic and histologic features of immune checkpoint inhibitor-related colitis. Inflamm Bowel Dis 2018;24:1695-1705.

66. Abu-Sbeih H, Ali FS, Naqash AR, et al. Resumption of immune checkpoint inhibitor therapy after immune-mediated colitis. J Clin Oncol 2019:37:2738-2745

67. Asher N, Marom EM, Ben-Betzalel G, et al. Recurrent pneumonitis in patients with melanoma treated with immune checkpoint inhibitors. Oncologist 2019;24:640-647.

68. Kikuchi H, Sakuraba H, Akemoto $Y$, et al. A case of nivolumab-associated colitis, which relapsed after mucosal healing and was then successfully treated with mesalazine. Immunol Med 2019;42:39-44. 
Supplemental online content for:

\section{Clinical Characteristics and Outcomes of Oral Mucositis Associated With Immune Checkpoint Inhibitors in Patients With Cancer}

Jake S. Jacob, MD; Barbara E. Dutra, MD; Victor Garcia-Rodriguez, MD; Kavea Panneerselvam, MD; Fiyinfoluwa O. Abraham, MD; Fangwen Zou, MD; Weijie Ma, MD; Petros Grivas, MD, PhD; John A. Thompson, MD; Mehmet Altan, MD; Isabella C. Glitza Oliva, MD, PhD; Hao Chi Zhang, MD; Anusha S. Thomas, MD; and Yinghong Wang, MD, PhD

J Natl Compr Canc Netw, 2021;19(12):1415-1424

eFigure 1: Patient Selection Criteria

eFigure 2: Overall Survival According to Mucositis Recurrence, Immunosuppressive Therapy, and Mucositis eTable 1: Clinical Characteristics and Outcomes of Mucositis eTable 2: Clinical Characteristics of Study Patients Based on Status of irAEs Other Than Mucositis 


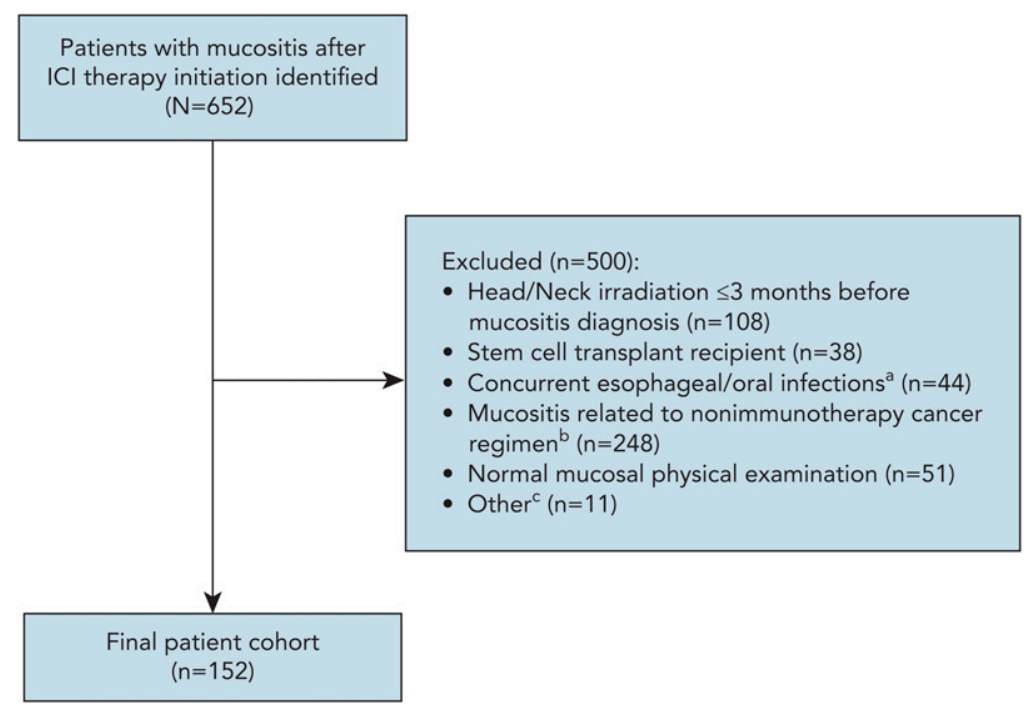

eFigure 1. Patient selection criteria.

Abbreviation: ICl, immune checkpoint inhibitor.

alncluding herpes simplex virus, cytomegalovirus, and Candida.

${ }^{\mathrm{b}}$ Chemotherapeutic agents, including alkylating agents, antitumor antibiotics, antimetabolites, vinca alkaloids, anthracyclines, and taxanes.

Including oral/tongue dysplasia, allergic reaction, mechanical trauma, and pill-induced sores. 
A

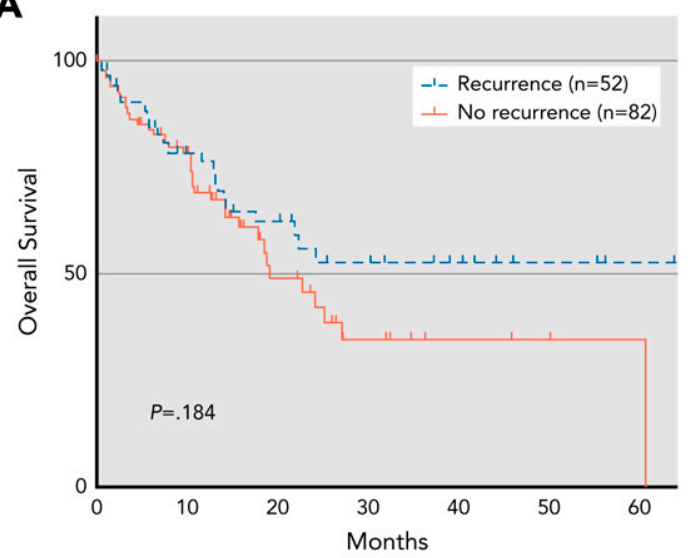

B

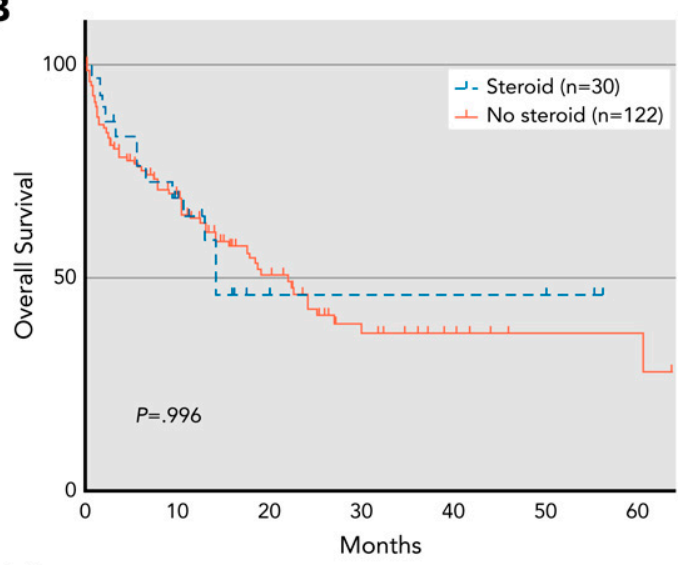

Number at risk

$\begin{array}{llllllll}\text { Steroid } & 30 & 18 & 5 & 4 & 4 & 4 & 1\end{array}$

$\begin{array}{llllllll}\text { No steroid } & 122 & 74 & 38 & 19 & 9 & 5 & 5\end{array}$

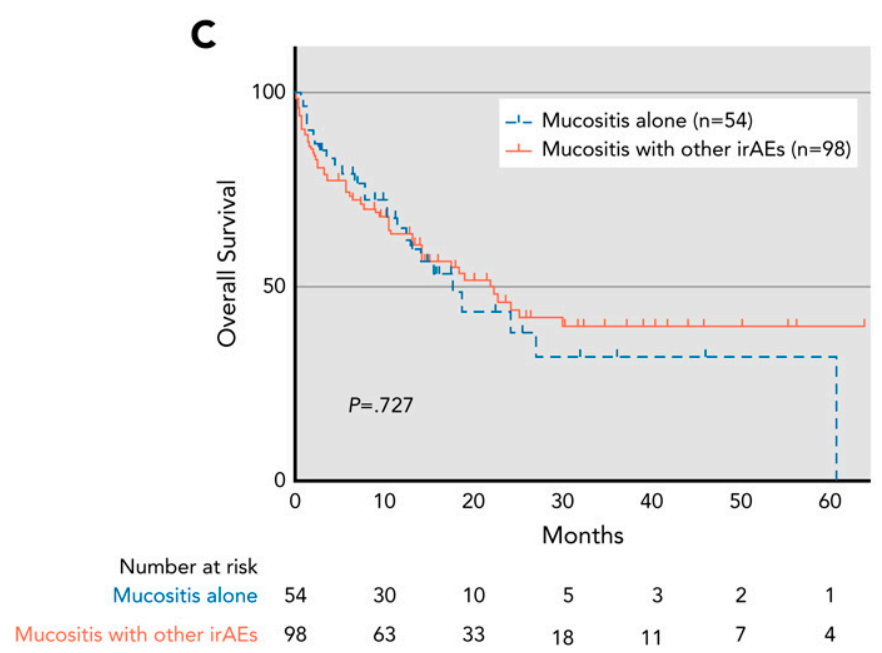

eFigure 2. Overall survival according to (A) mucositis recurrence, (B) immunosuppressive therapy, and (C) mucositis alone or with other irAEs. Abbreviation: irAE, immune-related adverse event. 


\section{eTable 1. Clinical Characteristics and Outcomes of Mucositis Stratified by Type of} Immunosuppressive Treatment

\begin{tabular}{|c|c|c|c|}
\hline Characteristic & $\begin{array}{l}\text { Systemic } \\
\text { Immunosuppressants } \\
\text { n (\%) }\end{array}$ & $\begin{array}{l}\text { Topical } \\
\text { Immunosuppressants } \\
\text { n (\%) }\end{array}$ & $P$ Value \\
\hline Total, $n$ & 29 & 7 & \\
\hline \multicolumn{4}{|l|}{ Clinical presentation } \\
\hline Odynophagia/Oral pain & $28(97)$ & $7(100)$ & 1.000 \\
\hline Nausea or vomiting & $6(21)$ & $0(0)$ & .451 \\
\hline Dysphagia & $5(17)$ & $0(0)$ & .565 \\
\hline Bleeding & $0(0)$ & $0(0)$ & \\
\hline Peak grade of mucositis & & & .710 \\
\hline $1-2$ & $25(86)$ & $7(100)$ & \\
\hline $3-4$ & $4(14)$ & $0(0)$ & \\
\hline Physical examination finding & & & 1.000 \\
\hline Ulcers/Aphthae & $28(97)$ & $7(100)$ & \\
\hline Nonulcer inflammation & $1(3)$ & $0(0)$ & \\
\hline \multicolumn{4}{|l|}{ Outcome } \\
\hline Hospitalization & $6(21)$ & $0(0)$ & .451 \\
\hline Median duration of hospitalization, $d(I Q R) ; n=29$ & $88(45-126)$ & $73(52-106)$ & .981 \\
\hline Clinical improvement/resolution & $26(90)$ & $7(100)$ & .899 \\
\hline Recurrence of mucositis & $15(52)$ & $5(71)$ & .822 \\
\hline All-cause mortality & $13(45)$ & $1(14)$ & .291 \\
\hline
\end{tabular}

Abbreviation: IQR, interquartile range. 
eTable 2. Clinical Characteristics of Study Patients Based on Status of irAEs Other Than Mucositis

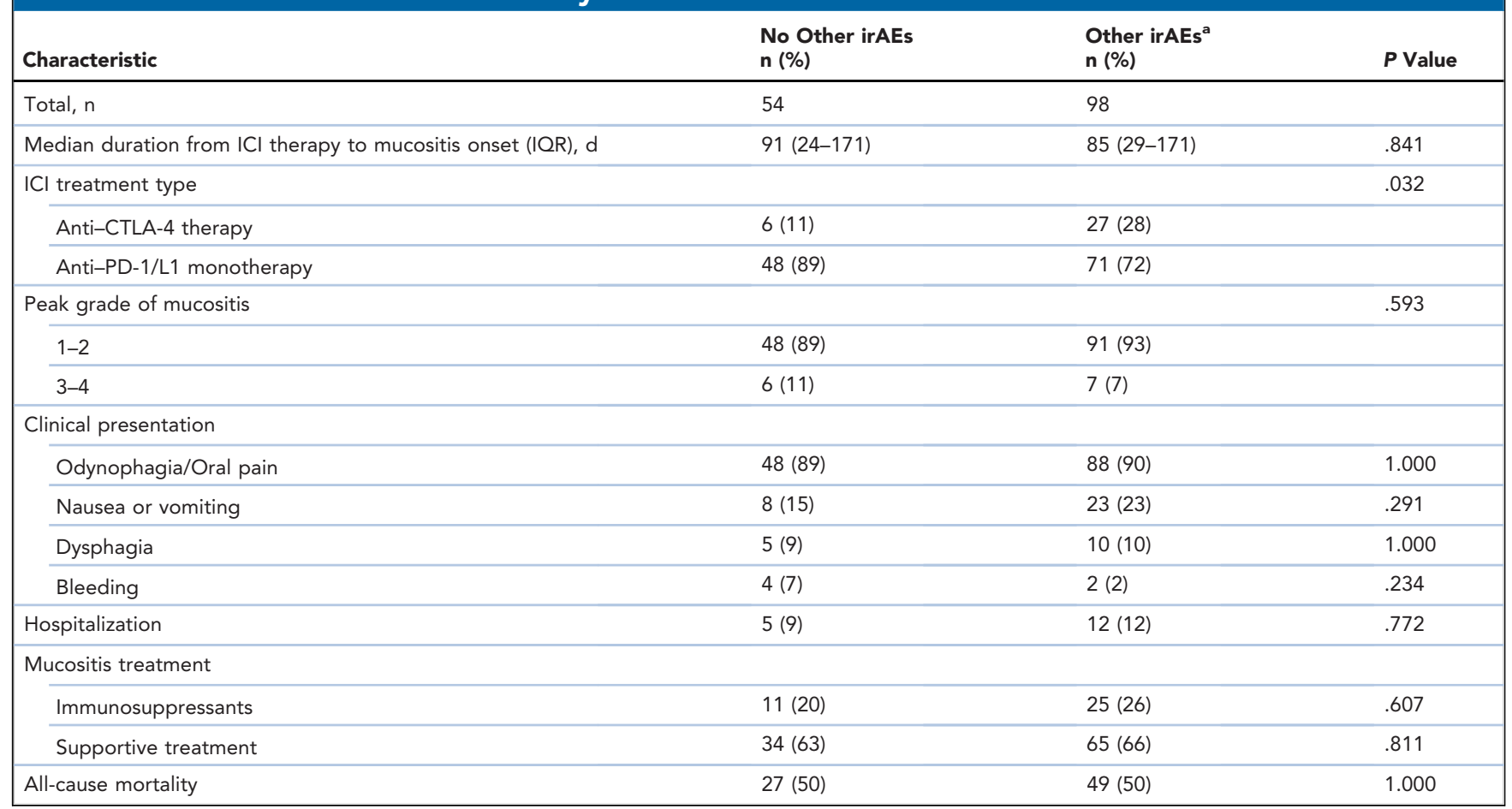

Abbreviations: $I C l$, immune checkpoint inhibitor; $I Q R$, interquartile range; irAE, immune-related adverse event.

ancluded hepatic, endocrine, pulmonary, dermatologic, hematologic, neurologic, renal, musculoskeletal, and ophthalmic toxicities. 